Aim of the study: In spite of the introduction of dose constraints based on patient individual assessment techniques for radiation therapy (RT), some side effects from the heart and lungs are observed. Regular physical exercises improve efficiency, which was confirmed in clinical trials. The aim of this study was to evaluate endurance exercise tolerance and the impact of aerobic training (AT) on selected clinical parameters in breast cancer patients during RT.

Material and methods: This study involved 46 women with breast cancer who were irradiated using conformal technique (3DCRT) to a total dose of 50 Gray during a 5-week course of RT. In this period 25 patients (group A) simultaneously performed AT, and the rest of the patients, without rehabilitation (group B), undertook irregular physical activity on their own. The exercise tolerance was assessed through the 6 -minute walk test (6MWT) using: oxygen saturation $\left(\mathrm{SO}_{2}\right)$, heart rate (HR), blood pressure (BP), 6-minute walk distance (6MWD) and dyspnea scale.

Results: After AT in group A, a statistically significant $(p<0.05)$ decrease was observed in average diastolic BP before 6MWT and in HR parameters before and after 6MWT $(p>0.05)$, and 6MWD was increased $(p<0.05)$. In group B, after RT, an increase $(p<0.05)$ in HR was observed after the test as well as dyspnea. Oxygen saturation in both groups was not significantly changed.

Conclusions: The results of our study showed that regular AT after just 6 weeks caused an improvement in exercise tolerance parameters with a substantial decline in dyspnea in breast cancer patients receiving RT.

Key words: neoplasm, rehabilitation, endurance, radiation, physical activity.

\section{Exercise tolerance in breast cancer patients during radiotherapy after aerobic training}

\author{
Piotr Milecki ${ }^{12}$, Katarzyna Hojan ${ }^{3}$, Owidia Ozga-Majchrzak³, \\ Marta Molińska-Glura ${ }^{4}$
}

1Department of Radiotherapy, Greater Poland Cancer Centre, Poznan, Poland ${ }^{2}$ Chair and Department of Electroradiology, Poznan University of Medical Sciences, Poland

${ }^{3}$ Department of Rehabilitation, Greater Poland Cancer Centre, Poznan, Poland ${ }^{4}$ Chair and Department of Computer Science and Statistics, Poznan University of Medical Sciences, Poland

\section{Introduction}

Physical activity (PA) can have a beneficial effect on both physical and mental health in cancer patients. However, evidence from cross-sectional and retrospective research suggests that cancer treatment can be associated with both short- and long-term reductions in PA [1-4]. While the benefit of postoperative radiotherapy (RT) for breast cancer is well documented [5, 6], there are a number of side effects associated with this treatment that might affect the patients' quality of life (QoL) and even possibly survival [7-9]. Among the long-term RT complications there is heart and lung damage, impaired shoulder mobility, and chronic pain [7, 8]. Many complications occur simultaneously during RT for breast cancer; therefore research on the impact of exercises and their effects is needed.

The purpose of this study was to examine whether moderate-intensity endurance training would have a positive effect on aerobic capacity (oxygen saturation, heart rate, blood pressure, dyspnea, walk distance) in comparison with those women who were not taking any PA during postoperative RT of breast cancer.

\section{Material and methods}

\section{Patients}

Forty-six patients with breast cancer from 37 to 65 years old were included in the study. The participants with breast cancer were recruited from the Outpatient Department of Radiotherapy of the Greater Poland Cancer Centre in Poznan. All study procedures were reviewed and approved by the Bioethics Committee at Poznan University of Medical Sciences, and participation in the study was conditional upon obtaining voluntary informed consent. All patients were recruited and randomly assigned to either an exercise or a control group. Some patients (group A) were simultaneously treated in the Rehabilitation Ward and received (for 6 weeks) AT. The rest of the patients, without rehabilitation (group B), took irregular PA on their own. Criteria for inclusion were: female gender, age between 20 and 65 years, histologically established breast cancer (AJCC stage I to stage IIIC), and good general health (ECOG performance status 0-1). The exclusion criteria included concurrent major health problems that could affect the patient's participation in an exercise program, including uncontrolled hypertension, cardiovascular diseases resulting in circulation failure (above stage II of heart failure according to the New York Heart Association), abnormal levels in blood tests, acute or chronic respiratory disease, and cognitive dysfunction as well as neuromuscular, musculoskeletal impairment or disease (except breast cancer) affecting motion pat- 
Table 1. Baseline characteristics in study groups (A and B)

\begin{tabular}{|c|c|c|c|}
\hline Parameters & Group A & Group B & $p$ \\
\hline number of patients & 35 & 31 & \\
\hline \multicolumn{4}{|c|}{ mean (SD } \\
\hline age [years] & $51.08(11.3)$ & $54(10.3)$ & 0.375 \\
\hline height [cm] & $165.12(6.7)$ & $162.95(5.6)$ & 0.255 \\
\hline body mass [kg] & $67.72(12.2)$ & $68.95(15.9)$ & 0.770 \\
\hline BMI & $24.88(4.6)$ & $25.82(5.1)$ & 0.843 \\
\hline \multicolumn{4}{|c|}{$\begin{array}{c}\text { Number of patients }-N \\
\text { Percentage of the whole study group (\%) }\end{array}$} \\
\hline \multicolumn{4}{|l|}{ side of the operated breast } \\
\hline right & 19 (54.3\%) & $16(45.7 \%)$ & \\
\hline left & $16(51.6 \%)$ & $15(48.3 \%)$ & \\
\hline \multicolumn{4}{|l|}{ surgical treatment: } \\
\hline breast-conserving therapy (BCT) & $24(68.6 \%)$ & $11(31.4 \%)$ & \\
\hline total mastectomy & $21(67.7 \%)$ & $10(32.2 \%)$ & \\
\hline \multicolumn{4}{|c|}{$\begin{array}{l}\text { clinical advancement degree } \\
\text { ( } \mathrm{T} \text { - tumor, } \mathrm{N} \text { - nodules, } \mathrm{M} \text { - metastases): }\end{array}$} \\
\hline $\mathrm{T}_{1} \mathrm{~N}_{0} \mathrm{M}_{0}$ & $3(8.6 \%)$ & $30(85.7 \%)$ & \\
\hline $\mathrm{T}_{1} \mathrm{~N}_{1} \mathrm{M}_{0}$ & $2(5.7 \%)$ & $2(6.4 \%)$ & \\
\hline $\mathrm{T}_{2} \mathrm{~N}_{1} \mathrm{M}_{0}$ & $24(77.4 \%)$ & $5(16.1 \%)$ & \\
\hline
\end{tabular}

tern or postural control. The baseline characteristics of both groups (A and B) are presented in Table 1.

\section{Interventions}

\section{Radiotherapy}

All participants in this study underwent breast surgery and then received external beam radiation treatments 7 days per week for 5 weeks. The affected breast and regional lymph nodes were treated to a total dose of 50 Gy in a daily fraction of 2 Gy after 4-5 weeks following surgical treatment [10]. Radiotherapy planning was performed using computed tomography to reduce radiation dose to the heart and lungs according to the dose constraints which were applied in each case. For patients after total mastectomy, chest wall and axillary lymph nodes were irradiated, and for women after breast-conserving surgery the entire breast was irradiated. Because of some differences in radiotherapy techniques, patients were stratified between arms with the aim of proper allocation.

\section{Exercise program}

Patients assigned to the exercise group attended a supervised exercise program 5 times per week for 6 weeks in the Rehabilitation Ward of the Greater Poland Cancer Centre in Poznan. Participation in the exercise group was voluntary, based on a referral from a radiation oncologist. The basic method of physiotherapy was endurance training by cycling.
The 40-45-minute exercises consisted of a 2-minute warmup, 40 minutes of cycling, and a 3-minute relaxation period. The PA was moderate with the maximal heart rate of $65-70 \%$ of the maximum (220-age). After cycling, group breathing exercises were introduced. They included respiratory muscle training, especially abdominal and diaphragm muscle workout, relaxation exercises and breathing exercises to increase respiratory motion in lower ribs and to teach diaphragmatic breathing. One or more physiotherapists assisted with all the exercises. The physical training was conducted before a radiotherapy session (after 23 hours following the previous radiotherapy dose).

\section{Outcome measures}

Testing was performed 1 week before (baseline) and 1 week after completion of RT.

\section{Six-minute walk test (6MWT)}

The 6MWT is a useful measure of functional capacity, targeted at people with at least moderately severe impairment. It has been widely used for measuring the response to therapeutic interventions for pulmonary and cardiac disease. The American Thoracic Society guidelines provide a standardized approach for performing the test $[11,12]$. The walk test was performed in an indoor corridor $30 \mathrm{~m}$ long. Patients were instructed to walk the corridor from one end to the other as many times as possible within the permitted time. The par- 
ticipants were instructed to come to the testing sessions wearing exercise clothing and comfortable shoes and to abstain from food and coffeine. The test was performed under the control of a physician who encouraged the patients with remarks such as "you are doing well". At the beginning and after 6 minutes the physician measured $\mathrm{SO}_{2}$, heart rate $(\mathrm{HR})$, arterial blood pressure including systolic blood pleasure (SBP) and diastolic blood pleasure (DBP) as well as the total distance walked (6-minute walk distance - 6MWD) by the patient. Arterial $\mathrm{SO}_{2}$ and $\mathrm{HR}$ can also be measured via pulse oximetry with a VM-2101-Finger Oximeter - Viamed, United Kingdom. Arterial blood pressure was measured on the arm on the side opposite to the breast cancer site. Secondary measures can include dyspnea measured with a modified Borg scale (0-10) [13, 14].

\section{Statistical analysis}

Data analyses were conducted using STATISTICA version 10.0, StatSoft Poland. The results of anthropometric parameter measures and 6MWT parameters were subjected to analysis. The quantitative data were described through mean, standard deviation, minimum and maximum. The Shapiro-Wilk test was used to assess the normal distribution compatibility. The differences between the results for $\mathrm{SO}_{2}, \mathrm{HR}$, SBP, DBP, $6 \mathrm{MWD}$ and speed were analyzed using the $t$-test for connected variables. To evaluate the subjective assessment concerning dyspnea, Wilcoxon's tests were used. The results with $p<0.05$ were regarded as statistically significant.

\section{Results}

Table 2 presents the list of mean and standard deviation values of $6 \mathrm{MWT}$ parameters and the $p$ value before $\mathrm{RT}$ in the study groups. There were no significant differences in the 6MWT parameters before the beginning of RT between the groups.

In group A falls in HR were found both before (1.3\%) and after (1.7\%) 6MWT (statistically insignificant), while a significant decrease in DBP before exercise was observed. After the end of the therapy in group A 6MWD $(p=0.00)$ significantly increased and hence so did walking speed. This group showed lower values than at the beginning of the assess- ments concerning dyspnea after RT but they were statistically insignificant.

In group B after RT a statistically significant rise in $\mathrm{HR}$ was observed both before and after 6MWT. Systolic blood pleasure was found to decrease by $1.9 \%$, including statistically significantly (6.7\%) after the test, and a small decrease in $6 \mathrm{MWD}$ (by 2.2\%) was observed. After RT the patients in group B reported greater dyspnea (by $29.5 \%$ ) than in group A - the differences were statistically significant.

Table 3 summarizes the parameters of $6 \mathrm{MWT}$ before and after RT and their statistical evaluation in the study groups.

\section{Discussion}

Many clinical data have confirmed the benefit in survival for postoperative RT in breast cancer patients [5-7]. However, there are a number of complications associated with this type of treatment. A common problem after RT for breast cancer is fatigue, depression, loss of physical functioning, and increased risk for side effects from heart, lung, skin, muscles and joints $[8,15,16]$. Physical activity during RT has a positive effect on most parameters in quality of life, dyspnea, and fitness (e.g. an increase in motion range in the shoulder) [1722]. In the present study we performed a trial to determine whether moderate-intensity aerobic exercise would offer some benefit to breast cancer patients during the course of RT by improving exercise capacity. We used the $6 \mathrm{MWT}$, which is the most common test for the objective assessment of exercise capacity in patients with cardiopulmonary disease [23]. This is a simple and widely used exercise tolerance measure in chronic respiratory diseases, heart failure as well as in cancer patients [23-26]. Miller et al. [25] found that the $6 \mathrm{MWT}$ might provide prognostic information beyond pulmonary function tests and dosimetric parameters in predicting RT-induced lung injury (RTLI). Rawat and co-authors [26] also confirmed the value of this test in assessing the risk of radiation-induced lung dysfunction in patients with cancer of the esophagus.

In the present study it was found that RT without regular PA changed 6MWT parameters. Especially a statistically significant increase in HR and SBP was noted. However, we did not find any significant effect of RT on 6MWD changes.

Table 2. List of mean and standard deviation values (SD) of 6MWT parameters and $p$ values before radiotherapy in study groups

\begin{tabular}{|lcccccc|}
\hline Parameters & Group A & Group B & $p$ & Group A & Group B & $p$ \\
\hline & & before 6MWT & & after 6MWT & \\
\hline $\mathrm{SO}_{2}[\%]$ & $98.37(0.9)$ & $98.45(0.7)$ & 0.583 & $98.05(1.1)$ & $98.15(0.8)$ & 0.717 \\
\hline $\mathrm{HR}[/ \mathrm{minute}]$ & $81.04(10.3)$ & $78.75(6.9)$ & 0.183 & $105.08(16.5)$ & $97.85(11.2)$ & 0.102 \\
\hline $\mathrm{SBP}[\mathrm{mm} \mathrm{Hg}]$ & $125.95(17.2)$ & $126.2(19.3)$ & 0.919 & $135.56(23.3)$ & $133.65(17.8)$ & 0.764 \\
\hline $\mathrm{DBP}[\mathrm{mm} \mathrm{Hg}]$ & $81.57(10.4)$ & $80.65(9.6)$ & 0.583 & $82.56(9.0)$ & $82.25(9.9)$ & 0.913 \\
\hline 6MWD $[\mathrm{m}]$ & - & - & - & $423.37(66.6)$ & $426.40(53.0)$ & 0.571 \\
\hline MET & - & - & - & $3.01(0.3)$ & $3.03(0.2)$ & 0.571 \\
\hline dyspnea & - & - & - & $3.48(1.4)$ & $3.05(1.9)$ & 0.305 \\
\hline
\end{tabular}

$p-p$ values, $6 M W T$ - 6-minute walk test, $\mathrm{SO}_{2}$-oxygen saturation, $\mathrm{HR}$ - heart rate, SBP-systolic blood pleasure, DBP-diastolic blood pleasure, GMWD - 6 minute walk distance, MET - metabolic equivalent of task 
Table 3. Summary of parameters of 6MWT before and after RT and their statistical evaluation

\begin{tabular}{|c|c|c|c|c|c|c|c|c|}
\hline \multirow[t]{2}{*}{ Parameters } & \multicolumn{4}{|c|}{ Group A } & \multicolumn{4}{|c|}{ Group B } \\
\hline & Before RT & After RT & Difference & $p$ & Before RT & After RT & Difference & $p$ \\
\hline \multicolumn{9}{|c|}{ before $6 \mathrm{MWT}$} \\
\hline $\mathrm{SO}_{2}[\%]$ & $98.37(0.8)$ & $98.22(0.9)$ & $-0.15(0.8)$ & 0.212 & $98.45(0.7)$ & 98.15 (1.0) & $-0.30(1.1)$ & 0.229 \\
\hline $\mathrm{HR}$ [/minutes] & $81.04(10.3)$ & $80.00(11.2)$ & $-1.04(10.1)$ & 0.494 & 78.75 (6.9) & 83.35 (11.1) & $4.60(8.6)$ & 0.012 \\
\hline $\mathrm{SBP}[\mathrm{mm} \mathrm{Hg}]$ & $125.95(17.2)$ & $123.62(15.8)$ & $-2.33(10.7)$ & 0.152 & $126.2(19.3)$ & $124.91(17.5)$ & $-1.35(11.2)$ & 0.598 \\
\hline $\mathrm{DBP}[\mathrm{mm} \mathrm{Hg}]$ & 81.57 (9.9) & $78.88(9.6)$ & $-2.68(7.1)$ & 0.044 & 80.65 (9.6) & $78.25(10.3)$ & $-2.40(6.7)$ & 0.126 \\
\hline \multicolumn{9}{|c|}{ after $6 \mathrm{MWT}$} \\
\hline $\mathrm{SO}_{2}[\%]$ & 98.08 (1.0) & $98.11(1.0)$ & $0.02(1.0)$ & 0.885 & $98.15(0.8)$ & 97.85 (1.0) & $-0.3(1.1)$ & 0.229 \\
\hline $\mathrm{HR}$ [/minutes] & $101.86(14.7)$ & $100.17(14.1)$ & $-1.68(12.8)$ & 0.381 & 97.85 (11.2) & $105.80(12.8)$ & 7.95 (10.1) & 0.002 \\
\hline $\mathrm{SBP}[\mathrm{mm} \mathrm{Hg}]$ & $134.71(20.9)$ & $126.24(16.8)$ & $-8.46(13.2)$ & 0.000 & 133.65 (17.8) & $126.90(18.1)$ & $-6.75(12.6)$ & 0.026 \\
\hline $\mathrm{DBP}[\mathrm{mm} \mathrm{Hg}]$ & $82.42(9.3)$ & 81.68 (10.6) & $-0.73(9.4)$ & 0.602 & $82.2(9.8)$ & $83.20(13.2)$ & 0.95 (10.9) & 0.701 \\
\hline $6 \mathrm{MWD}[\mathrm{m}]$ & $423.37(66.6)$ & $447.33(65.3)$ & 23.95(36.1) & 0.000 & $426.40(53.0)$ & $417.00(57.6)$ & $-9.40(32.6)$ & 0.213 \\
\hline Speed $[\mathrm{km} / \mathrm{h}]$ & $4.23(0.6)$ & $4.45(0.6)$ & $0.24(0.3)$ & 0.000 & $4.26(0.5)$ & $4.17(0.6)$ & $-0.09(0.3)$ & 0.213 \\
\hline MET & $3.01(0.3)$ & $3.13(0.3)$ & $0.11(0.2)$ & 0.000 & $3.03(0.2)$ & $2.98(0.3)$ & $-0.04(0.1)$ & 0.213 \\
\hline dyspnea & $3.48(1.4)$ & $2.96(1.3)$ & $-0.51(0.7)$ & 0.764 & 3.05 (1.9) & $3.95(1.5)$ & $0.91(0.7)$ & 0.018 \\
\hline
\end{tabular}

$R T$ - radiotherapy; $p$ - $p$ values; $6 M W T$ - 6-minute walk test; $S_{2}$-oxygen saturation; HR - heart rate; SBP - systolic blood pleasure; DBP-diastolic blood pleasure; 6MWD - 6-minute walk distance; MET - metabolic equivalent of task

As Rawat [26] showed, the changes in the distance correlate with those in pulmonary function tests (PFTs). In the group without regular PA a decrease in the distance was found in $6 \mathrm{MWT}$ but it was not substantial. In the aerobic exercise group during RT, after the end of the therapy, there were found improvements of HR (both resting and post-activity), SBP as well as 6MWD. The study found that the women who performed moderate-intensity aerobic exercise during RT of breast cancer were able to prevent declines in aerobic capacity. The results of our study are consistent with those presented by Mustian et al. [21]. This improvement of aerobic capacity may be due to the changes in erythrocyte levels in the aerobic exercise group during radiation treatment of breast cancer, as observed by Drouin and co-authors [22]. As far as the fatigue intensification assessment after RT is concerned, our results confirm other authors' observations [19, 21]. The moderate-intensity aerobic training caused dyspnea to decrease, which emphasizes the significant physiotherapy value during RT in this group of patients [20, 21]. The study results support the potential for $\mathrm{PA}$ to be a safe, effective, and economical method for improving aerobic fitness in females undergoing radiation treatment of breast cancer. However, any factors could play an additional role in maintaining proper psycho-physical status of patients who undergo oncological therapy [27].

The results of the study suggest that moderate-intensity aerobic exercise performed during radiation treatment may preserve or maintain capacity tolerance parameters with a substantial change in dyspnea in females being treated for breast cancer compared with declines observed in non-training peers.

The authors declare no conflicts of interest.

\section{References}

1. Galvăo DA, Newton RU. Review of exercise intervention studies in cancer patients. J Clin Oncol 2005; 23: 899-909.

2. Knols R, Aaronson NK, Uebelhart D, Fransen J, Aufdemkampe G. Physical exercise in cancer patients during and after medical treatment: a systematic review of randomized and controlled clinical trials. J Clin Oncol 2005; 23: 3830-42.

3. Stevinson C, Lawlor DA, Fox KR. Exercise interventions for cancer patients: systematic review of controlled trials. Cancer Causes Control 2004; 15: 1035-56.

4. Courneya KS, Friedenreich CM. Physical exercise and quality of life following cancer diagnosis: a literature review. Ann Behav Med 1999; 21: 171-9.

5. Donovan E, Bleakley N, Denholm E, et al. Randomised trial of standard 2D radiotherapy (RT) versus intensity modulated radiotherapy (IMRT) in patients prescribed breast radiotherapy. Radiother Oncol 2007; 82: 254-64.

6. Rutqvist LE, Rose C, Cavallin-Stíhl E. A systematic overview of radiation therapy effects in breast cancer. Acta Oncol 2003; 42: 532-45.

7. Favourable and unfavourable effects on long-term survival of radiotherapy for early breast cancer: an overview of the randomised trials. Early Breast Cancer Trialists' Collaborative Group. Lancet 2000; 355: 1757-70.

8. Senkus-Konefka E, Jassem J. Complications of breast-cancer radiotherapy. Clin Oncol (R Coll Radiol) 2006; 18: 229-35.

9. Ma J, Zhang J, Zhou S, et al. Association between RT-induced changes in lung tissue density and global lung function. Int J Radiat Oncol Biol Phys 2009; 74: 781-9.

10. Cerezo L, Maciŕ M. Acute radiation syndrome and Fukushima: a watershed moment? Rep Pract Oncol Radiother 2012; 17: 1-3.

11. ATS statement: guidelines for the six-minute walk test. ATS Committee on Proficiency Standards for Clinical Pulmonary Function Laboratories. Am J Respir Crit Care Med 2002; 166: 111-117.

12. Solway S, Brooks D, Lacasse $Y$, Thomas S. A qualitative systematic overview of the measurement properties of functional walk tests used in the cardiorespiratory domain. Chest 2001; 119: 256-70.

13. Mancini I, Body JJ. Assessment of dyspnea in advanced cancer patients. Support Care Cancer 1999; 7: 229-32. 
14. Wilson RC, Jones PW. A comparison of the visual analogue scale and modified Borg scale for the measurement of dyspnoea during exercise. Clin Sci (Lond) 1989; 76: 277-82.

15. Magnan MA, Mood DW. The effects of health state, hemoglobin, global symptom distress, mood disturbance, and treatment site on fatigue onset, duration, and distress in patients receiving radiation therapy. Oncol Nurs Forum 2003; 30: 33-9.

16. Geinitz H, Zimmermann FB, Stoll P, et al. Fatigue, serum cytokine levels, and blood cell counts during radiotherapy of patients with breast cancer. Int J Radiat Oncol Biol Phys 2001; 51: 691-8.

17. Cadmus LA, Salovey P, Yu H, Chung G, Kasl S, Irwin ML. Exercise and quality of life during and after treatment for breast cancer: results of two randomized controlled trials. Psychooncology 2009; 18: 343-52.

18. Oliveira MM, Souza GA, Miranda Mde S, Okubo MA, Amaral MT, Silva MP, Gurgel MS. Upper limbs exercises during radiotherapy for breast cancer and quality of life. Rev Bras Ginecol Obstet 2010; 32: 133-8.

19. Hwang JH, Chang HJ, Shim YH, Park WH, Park W, Huh SJ, Yang JH. Effects of supervised exercise therapy in patients receiving radiotherapy for breast cancer. Yonsei Med J 2008; 49: 443-50.

20. Windsor PM, Nicol KF, Potter J. A randomized, controlled trial of aer obic exercise for treatment-related fatigue in men receiving radical external beam radiotherapy for localized prostate carcinoma. Cancer 2004; 101: 550-7.

21. Mustian KM, Peppone L, Darling TV, Palesh O, Heckler CE, Morrow GR. A 4-week home-based aerobic and resistance exercise program during radiation therapy: a pilot randomized clinical trial. J Support Oncol 2009; 7: 158-67.

22. Drouin JS, Young TJ, Beeler J, Byrne K, Birk TJ, Hryniuk WM, Hryniuk LE. Random control clinical trial on the effects of aerobic exercise training on erythrocyte levels during radiation treatment for breast cancer. Cancer 2006; 107: 2490-2495.

23. Salzman SH. The 6-min walk test: clinical and research role, technique, coding, and reimbursement. Chest 2009; 135: 1345-52.

24. Mao J, Zhang J, Zhou S, Das S, Hollis DR, Folz RJ, Wong TZ, Marks LB. Updated assessment of the six-minute walk test as predictor of acute radiation-induced pneumonitis. Int J Radiat Oncol Biol Phys 2007 67: 759-67.

25. Miller KL, Kocak Z, Kahn D, et al. Preliminary report of the 6-minute walk test as a predictor of radiation-induced pulmonary toxicity. Int J Radiat Oncol Biol Phys 2005; 62: 1009-13.

26. Rawat S, Kumar G, Puri A, et al. Correlation of six-minute walk test, pulmonary function test and radiation pneumonitis in the management of carcinoma of oesophagus: A prospective pilot study. J Radioth in Pract 2011; 10: 191-9.

27. Bogaczewicz J, Sysa-Jędrzejowska A, Karczmarewicz E, et al. Analysis of the dynamics of vitamin D status in the population of the Lodz region - a preliminary report. Post Dermatol Alergol 2011; 3: 170-4.

\section{Address for correspondence}

Katarzyna Hojan, MD, PhD

Department of Rehabilitation

Greater Poland Cancer Center

15 Garbary Street,

61-866 Poznan, Poland

tel. +48618850705

$\mathrm{fax}+48618521948$

e-mail: khojan@op.pl

Submitted: 10.02 .2013

Accepted: 28.03.2013 